\title{
Una mirada a la nueva Guía de Prevención de Infecciones en el área de anestesia en el quirófano
}

\section{A look at the new expert guidance for infection prevention in the anesthesia work area}

Hilda Guadalupe Hernández-Orozco, ${ }^{1}$ Gonzalo Bearman ${ }^{2}$

\section{INTRODUCCIÓN}

Se estima que en Estados Unidos cada año suceden 157,000 infecciones del sitio quirúrgico asociadas con la atención de la salud, ${ }^{1}$ con 8205 muertes vinculadas con ellas. También se atribuye que $11 \%$ de las muertes en unidades de cuidados intensivos se relacionan con infecciones del sitio quirúrgico. ${ }^{2}$

Las infecciones del sitio quirúrgico aumentan la estancia hospitalaria de pacientes 11 días en promedio ${ }^{3}$ y ocasionan $20 \%$ de los reingresos del paciente no planeados. ${ }^{4} \mathrm{El}$ costo de atención de estas infecciones alcanza 3200 millones de dólares anuales. ${ }^{3}$ En los últimos 5 años, en el Instituto Nacional de Pediatría las infecciones de todos los servicios quirúrgicos reportan tasas mínimas de 5.6 (en 2016) a máxima 8.4 (en 2018) por 1000 días paciente. ${ }^{5}$

Lo anterior denota la importancia del problema de las infecciones del sitio quirúrgico y la necesidad de la búsqueda de estrategias de prevención; secundario a ello se ha explorado la participación del proceso de anestesia como un factor contribuyente para la generación de estas infecciones.

\section{El problema}

La evidencia demuestra que las cinco superficies más contaminadas en el quirófano son el teclado de la enfermera, el teclado y ratón del anestesiólogo, el cajón del carro de anestesia y la mesa de operaciones. El grado de contaminación observado en el área de anestesia indica que aun cuando los anestesiólogos no tienen contacto directo con el sitio quirúrgico, su potencial contribución a la trasmisión de infecciones

\footnotetext{
${ }^{1}$ Departamento de Infectología

Comité de Infecciones Asociadas con la Atención de la Salud Instituto Nacional de Pediatría

${ }^{2}$ Hospital Epidemiologist

Virginia Commonwealth University

Correspondence

Hilda Guadalupe Hernández-Orozco wuzhi1916@gmail.com

Gonzalo Bearman

gonzalo.bearman@vcuhealth.org

Este artículo debe citarse como Hernández Orozco HG, Bearman Gonzálo. Una mirada a la nueva Guía de Prevención de Infecciones en el área de anestesia en el quirófano. Acta Pediatr Méx 2019;40(4):181-190.
} 
durante los procedimientos es alto; por lo que se desarrollan un conjunto de consideraciones para la prevención de infecciones en esta área: manipulación y limpieza de equipos, seguir una técnica aséptica, administración segura de medicamentos, y la medida más simple y efectiva: practicar la adecuada higiene de manos. Se reporta que de ésta, los anestesiólogos sólo cumplen $20 \%$ de la higiene de manos que deberían realizar y usan guantes cuando está indicado en $10 \%$ de los casos. También Ilevan a cabo acciones de riesgo : manipular equipo multiusos, medicamentos multidosis e inconsistencia en la asepsia del punto de inyección de fármacos en el equipo. Otro factor de riesgo durante la cirugía es que en algunas ocasiones el anestesiólogo manipula la posición del paciente a petición del cirujano tocando superficies fuera de su área de anestesia. ${ }^{6}$

La problemática de prevención en el trabajo diario del anestesiólogo se documenta al determinar que para llevar a cabo el proceso de anestesia del paciente debería lavarse las manos al menos 54 veces por hora y esto influye en la no ejecución de esta medida. Se ha observado un cumplimiento de sólo 17\% de apego a la higiene de manos por parte del anestesiólogo. Algunos investigadores mencionan que lo anterior es imposible, por lo menos en los 5 momentos durante el proceso de inducción anestésica. Otro factor contribuyente es el volumen de trabajo acelerado, que en ocasiones implica la atención de un nuevo procedimiento anestésico en los siguientes 10 a 15 minutos, lo que es un desafío para la práctica de la higiene de manos como para llevar a cabo la limpieza del equipo de anestesia, del carro de anestesia, y del ambiente alrededor de éste; sin embargo, aun así deben efectuarse estas medidas de prevención para evitar la trasmisión cruzada de microorganismos y la generación de riesgos de infección asociada con la atención de la salud. ${ }^{7}$
En el proceso de intubación endotraqueal o el manejo de la vía aérea que realiza el anestesiólogo, las manos pueden contaminarse con secreciones de la vía aérea superior y, secundariamente, ocurre la contaminación cruzada del área de trabajo de anestesia. Maslyk PA y su grupo $^{8}$ encontraron que el área de trabajo de anestesia estaba contaminada con carga biológica de bacterias comensales y patógenas, incluidos: estafilococos coagulasa negativos, $\mathrm{Ba}$ cillus spp, estreptococos, Staphylococcus aureus, Acinetobacter spp, y otras bacterias gramnegativas. Mahida y colaboradores encontraron durante la anestesia de pacientes que, de 426 jeringas usadas, $15 \%$ de las puntas de éstas y $4 \%$ de los contenidos tenían crecimiento bacteriano, predominantemente bajos recuentos de colonias de microorganismos de la piel (Staphylococcus spp coagulasa negativa, Micrococcus y Kocuria).

La contaminación del contenido de la jeringa fue significativamente más frecuente durante las cirugías de urgencia que en las electivas (razón de momios, $4.5 ; \mathrm{p}=0.01$ ).

Loftus y sus coautores ${ }^{9}$ estudiaron la repercusión de la contaminación de las llaves de paso con las bacterias que contaminan las manos de los anestesiólogos en el área hospitalaria y encontraron que las manos y el ambiente ocasionaban trasmisión cruzada a las llaves de paso, que se asoció con aumento de la mortalidad del paciente a los 30 días. Henry B y su grupo reportaron, en 1999, un brote de Serratia marcescens en 7 pacientes posoperados en quienes identificaron como factor de riesgo a un solo anestesiólogo que preparó simultáneamente múltiples jeringas de propofol sin usar guantes para extraer la sustancia de las jeringas o para realizar las intubaciones. ${ }^{10}$

La superficie de trabajo de la máquina de anestesia, controles de flujo de gas, diales del vaporizador, válvula limitadora de presión ajus- 
table (APL), soportes intravenosos, calentadores de fluidos, carrito de suministros, teclado de computadora, el ratón, son también ejemplos de componentes que se contaminan en el área de anestesia. Existen brotes que se asocian con el uso de laringoscopios contaminados.

Por lo que se refiere a los laringoscopios, el estudio de Lowman y su grupo ${ }^{11}$ encontró la contaminación con bacterias viables incluso en $57 \%$ de las cuchillas y $86 \%$ de los mangos de los laringoscopios que fueron desinfectados y estaban listos para su uso en el próximo paciente. Bhatt y colaboradores ${ }^{12}$ también encontraron contaminación bacteriana de laringoscopios de fibra óptica flexibles.

El personal de anestesia expresó las razones para no llevar a cabo la higiene de manos en una encuesta efectuada en 396 médicos anestesiólogos y 246 enfermeras anestesistas a quienes se mencionó que las causas son la falta de tiempo por situaciones de urgencia (58.3\%), falta de tiempo en general $(44.2 \%)$, factores de la piel $(35.8 \%)$, infraestructura o contenedores para higiene de manos no accesibles (44.2\%) y falta de apoyo del personal de quirófano para interrupciones en el flujo de trabajo relacionado con la higiene de manos $(15.5 \%)$. Se refirieren diferentes porcentajes de apego a la higiene de manos en los hospitales en anestesiólogos que van desde la ausencia de ésta, $24.25,34.9$ y $40.9 \%$ y el cumplimiento de otras medidas de prevención: 20.9 a $42.3 \% .^{13}$

En cuanto a la instalación de catéteres venosos centrales los anestesiólogos identificaron como medidas de barrera para efectuar el proceso adecuadamente, el uso de: mascarilla quirúrgica (94.4\%), de guantes estériles $(93.8 \%)$, de bata (88\%), de gorro $(91.6 \%)$ y de campos completos (79.2\%). La práctica fue diferente para las líneas arteriales porque las medidas de barrera se utilizaban en menor proporción: mascarillas quirúrgicas (82\%), guantes estériles $(74.2 \%)$, bata $(10.9 \%)$ y campos (3.7\%). ${ }^{14,15}$

\section{Recomendaciones de la guía}

En el Cuadro 1 se muestran las recomendaciones a que llegaron los autores. Todos son miembros de la Sociedad de Epidemiología Hospitalaria de América (SHEA) y representantes de organizaciones asociadas como la Sociedad Americana de Anestesiología (ASA), la Fundación de Seguridad de Pacientes de Anestesia (APSF) y la Asociación Americana de Enfermeras de Anestesia (AANA). Para documentar las recomendaciones aquí mostradas los autores realizaron una revisión exhaustiva de la bibliografía y levantaron encuestas con enfermeras y médicos encargados del proceso de anestesia. ${ }^{7}$

Muñoz-Price $S$ y sus colegas ${ }^{7}$ prepararon una guía de prevención de infecciones en el área de anestesia. La desarrollaron con base en la estrategia PICO buscando los estudios de acuerdo con el problema, las intervenciones, análisis y control que pudiera solucionarlo. Los comensales comunes fueron los microorganismos más frecuentemente aislados (79.1\%), pero se aislaron patógenos hospitalarios importantes: Enterobacter sp y Acinetobacter baumannii. El panel identificó las preguntas utilizadas en el desarrollo de términos de búsqueda (título del tema médico [MeSH] y palabra de texto), y se votaron tanto las preguntas como los términos de búsqueda por el panel de expertos hasta lograr la aprobación unánime. El panel identificó el periodo del 1 de enero de 1990 al 30 de junio de 2016 para los artículos estilo PICO; este sistema consiste en una iniciativa internacional para optimizar la evaluación de la calidad de la evidencia, calificando la fuerza de las recomendaciones que mejora la trazabilidad y la transparencia del proceso. ${ }^{7}$

\section{CONCLUSIÓN}

De acuerdo con la Guía, se necesitan más estudios de investigación para determinar los factores 


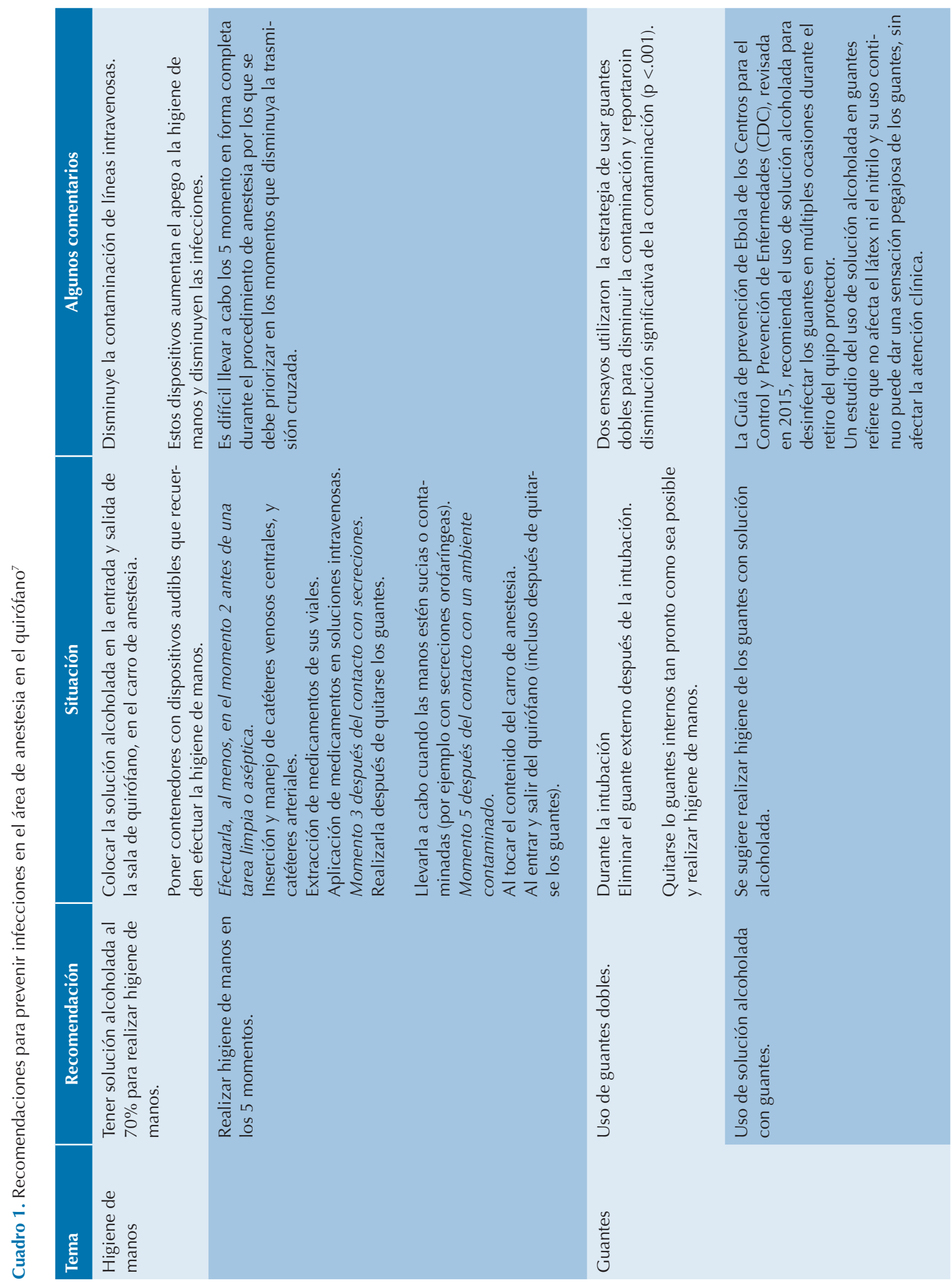




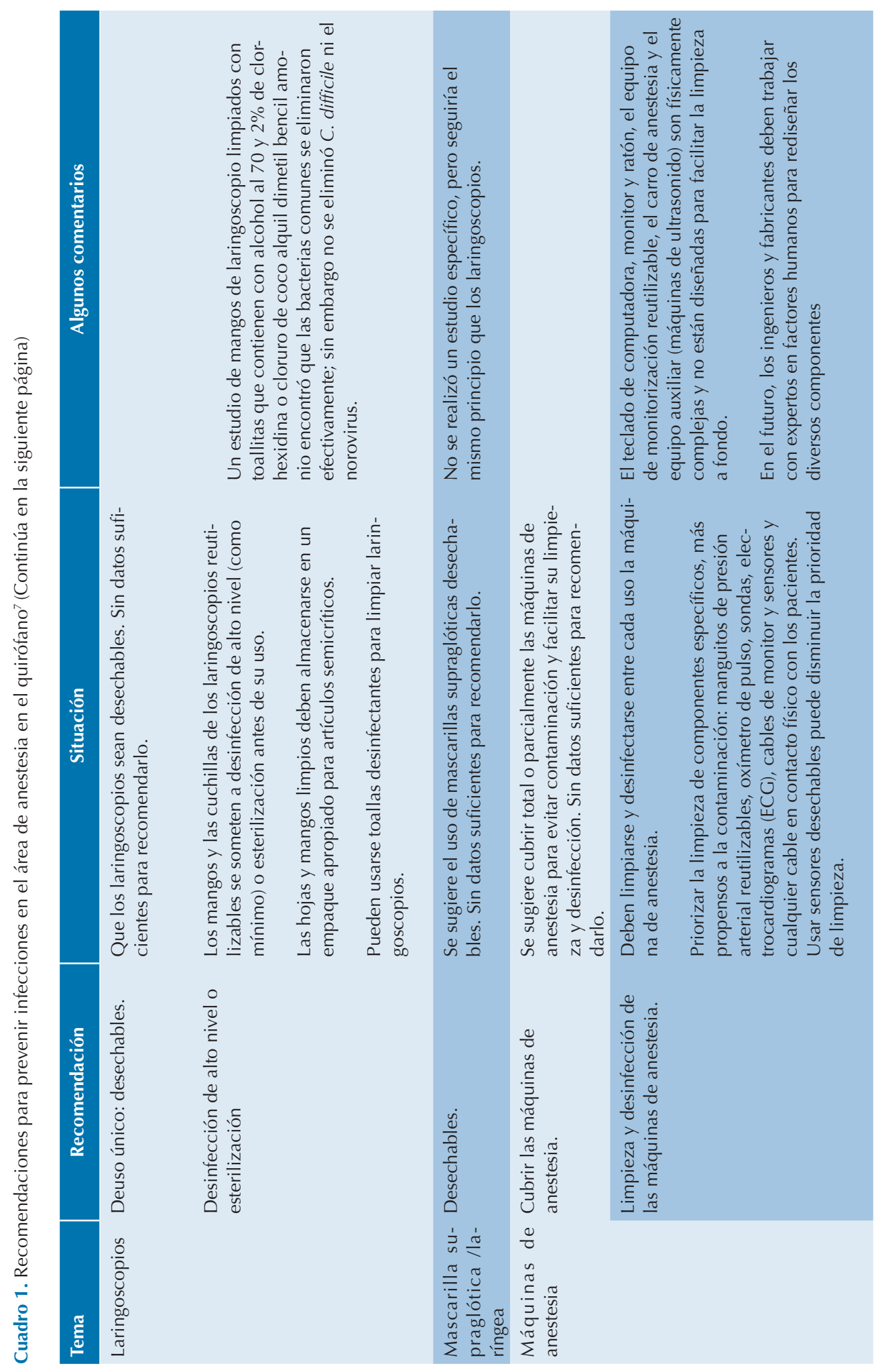




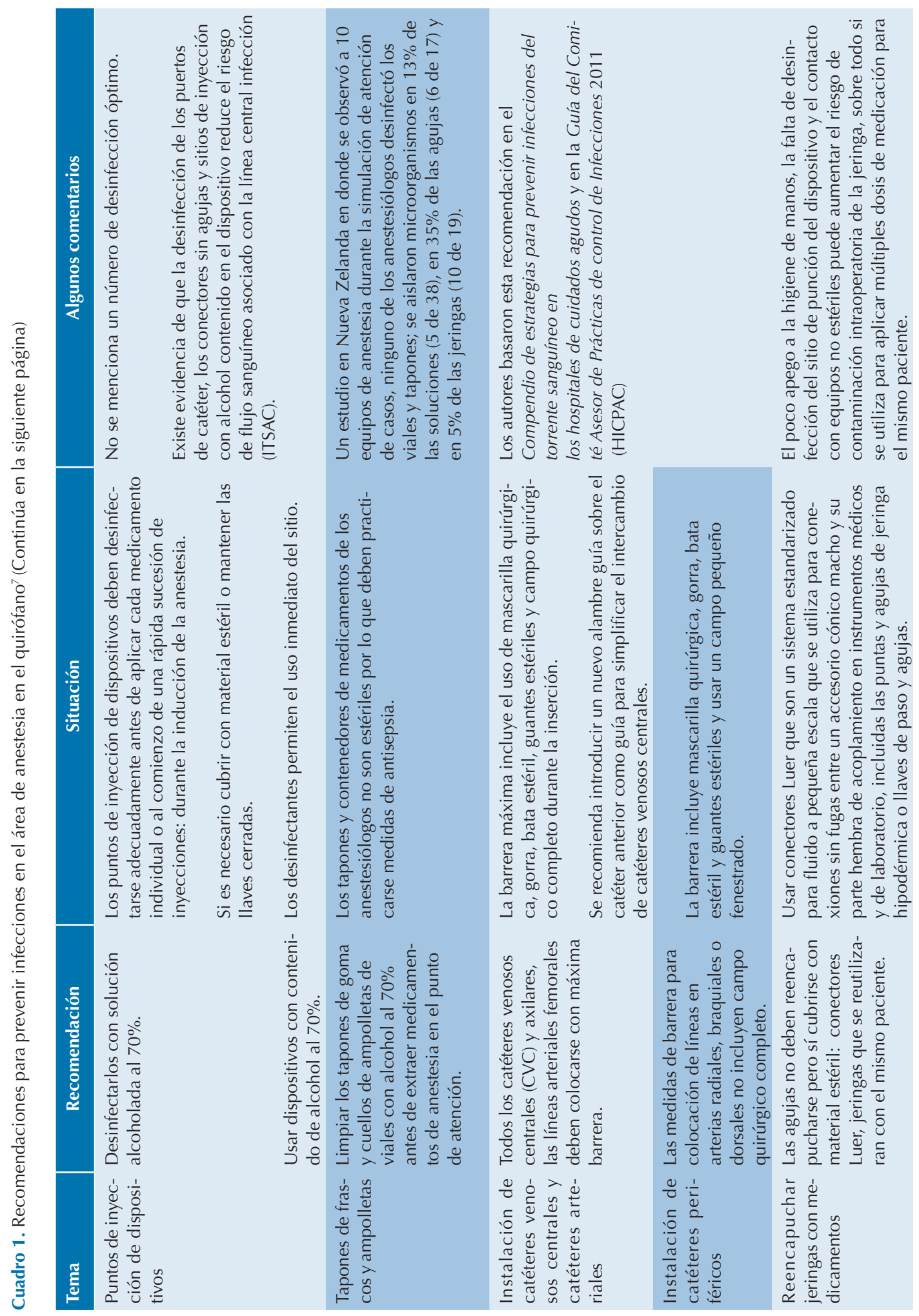




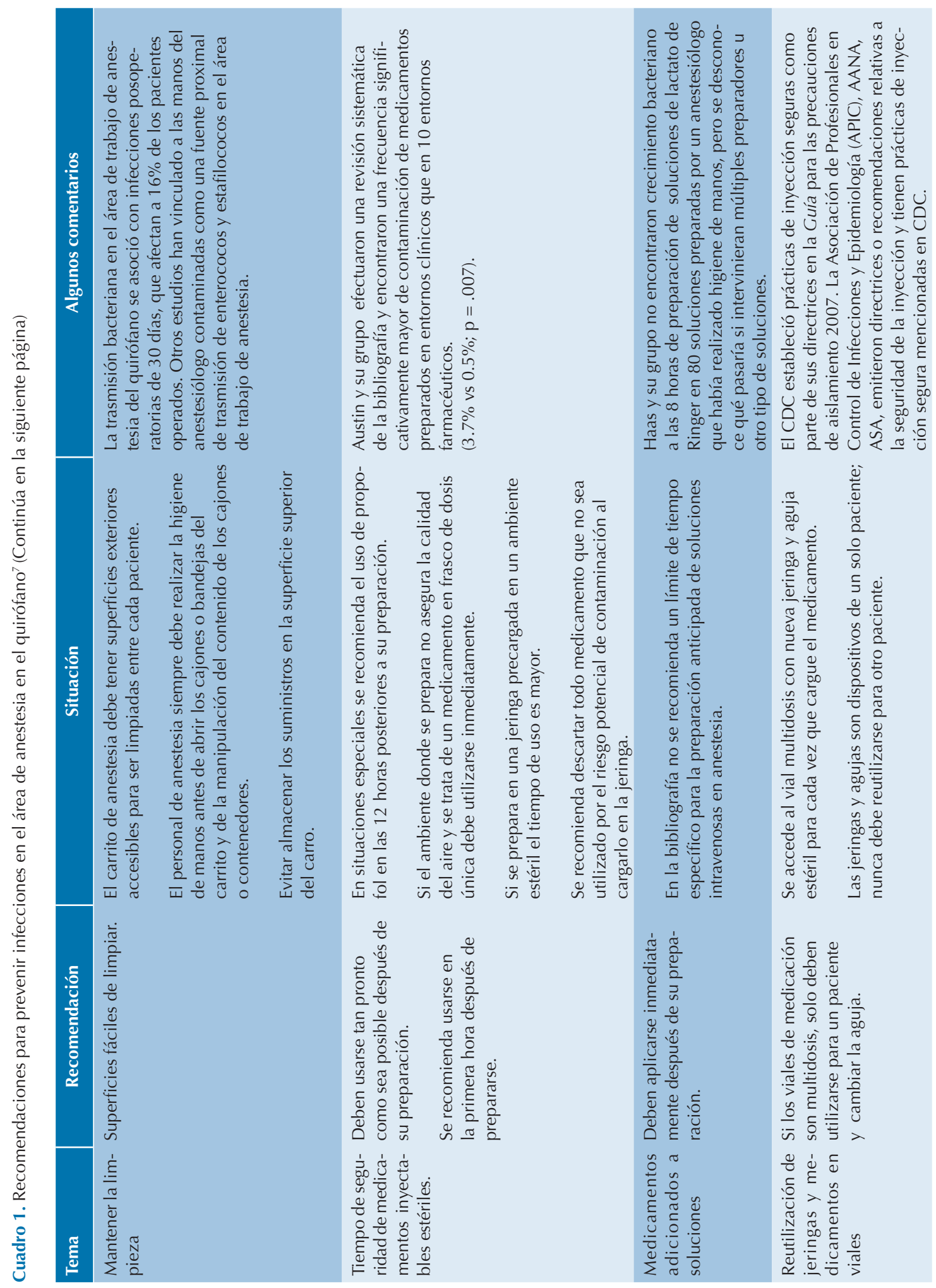




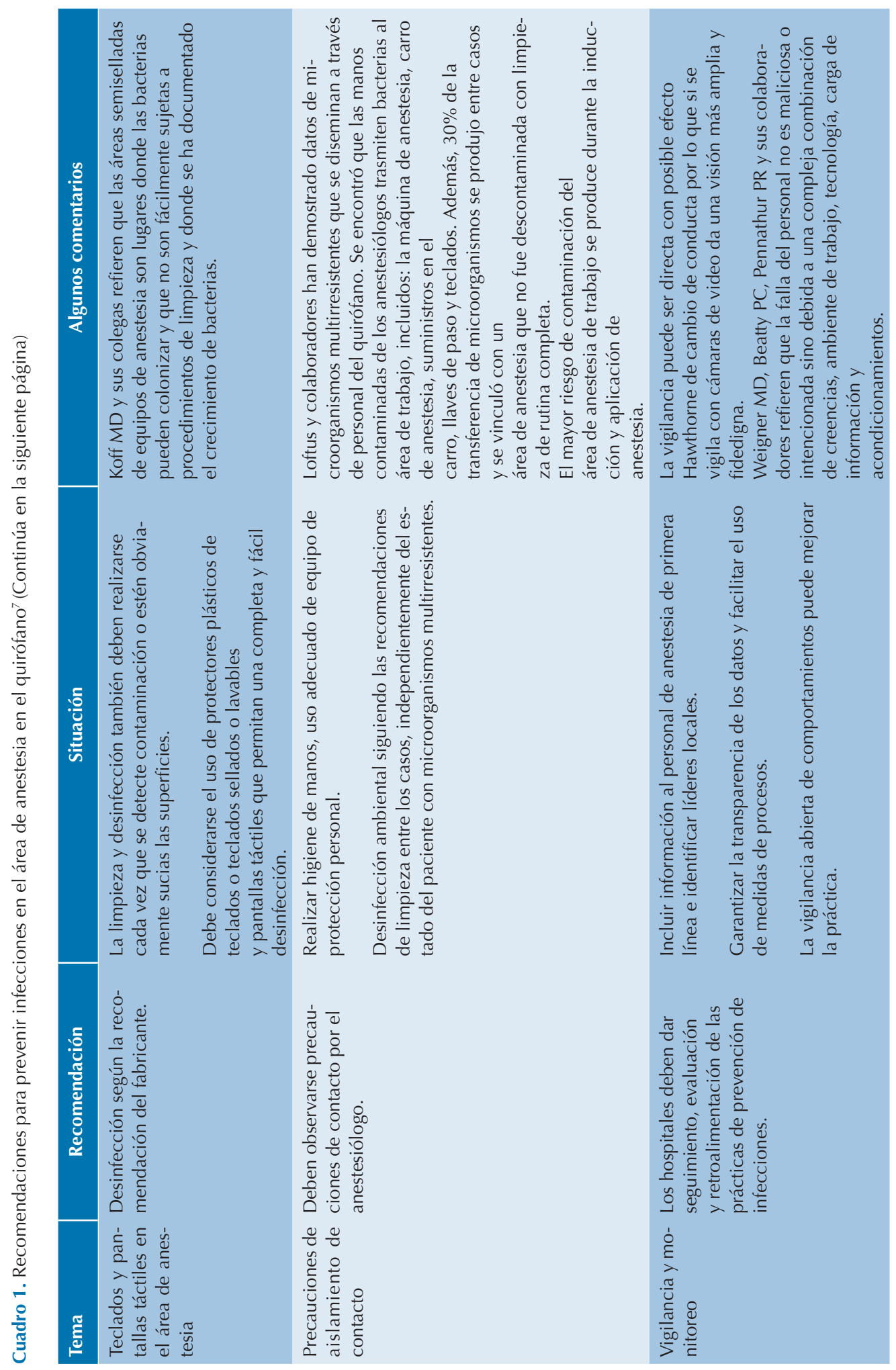




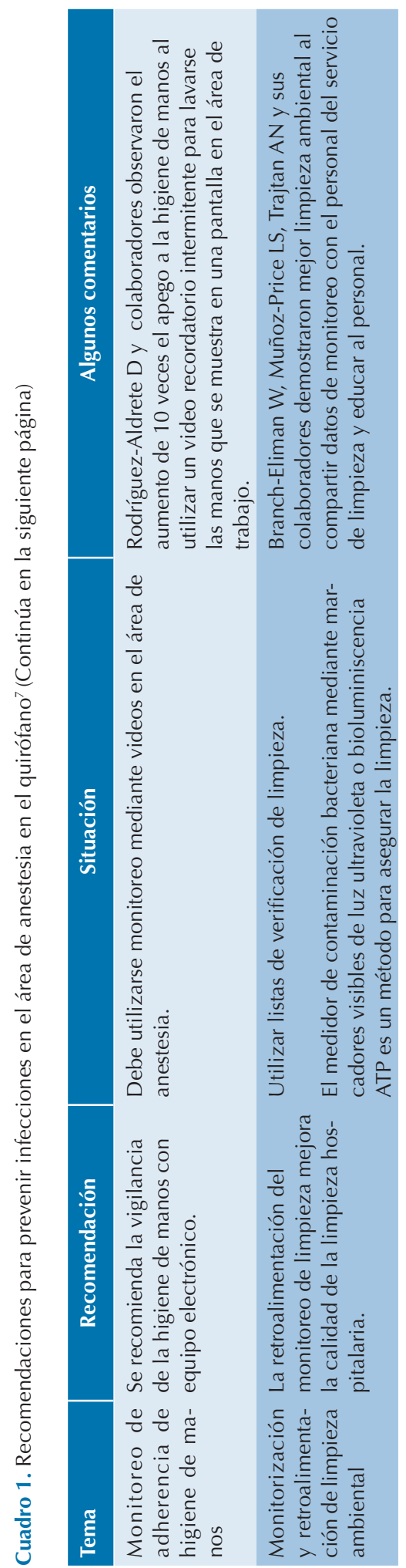

de riesgo asociados con el proceso de anestesia y la generación de infecciones asociadas con la atención de la salud, y para planear estrategias de prevención de acuerdo con los resultados de los factores identificados. Algunas recomendaciones emitidas por la guía refieren no tener evidencia científica contundente, como el uso de doble guante por el anestesiólogo. Como medidas de prevención para disminuir la trasmisión cruzada, en la realización del trabajo del anestesiólogo, debido a la problemática de realizar higiene de manos con la frecuencia requerida, se propone conocer esta Guía y aplicar las medidas ahí recomendadas para disminuir el riesgo de generación de infecciones asociadas con el proceso anestésico, darles seguimiento y evaluar las que aún no tienen fuerte evidencia para mejorar su aplicabilidad mediante la evaluación de su factibilidad y eficacia.

Otras de las medidas recomendadas ya han sido probadas como efectivas en la prevención de infecciones asociadas con la atención de la salud, como la higiene de manos y sólo se requieren estrategias para reforzar su cumplimiento por el anestesiólogo. Lo más importante es la difusión de estas guías y su puesta en práctica en los hospitales con el objetivo de utilizar una herramienta más para la disminución de infecciones asociadas con la atención de la salud durante la cirugía.

\section{REFERENCIAS}

1. Magill SS, et al. Multistate point-prevalence survey of health care-associated infections. N Engl J Med 2014;370(13):1198-208. doi: 10.1056/NEJMoa1306801

2. KlevensRM, Edwards JR, et.al. Estimating healthcareassociated infections and deaths in U.S. Hospitals, 2002. Public Health Reports 2007; 122:160-66. https://doi. org/10.1177/003335490712200205

3. ZimlichmanE, Henderson D, et al. Health care-associated infections: a meta-analysis of costs and financial impact on the US health caresystem. JAMA Intern Med. 2013; 173(22):2039-46. doi:10.1001/jamainternmed.2013.9763

4. MerkowRP, et al. Underlying reasons associated with hospital readmission following surgery in the United States JAMA. 2015;313(5):483-95. doi:10.1001/jama.2014.18614 
5. Informes del Comité de Infecciones Asociadas a la Atención de la Salud. Instituto Nacional de Pediatría 2015-2018. Literatura gris no publicada.

6. 6. Franklin E, Stein L. Human Factors Analysis of Infection prevention practices in the anesthesia work environment. Proceedings of the Human Factors and Ergonomics Society Annual Meeting 2017;61(1):639-42. https://doi. org/10.1177/1541931213601644

7. Munoz-Price SL, et al. Infection prevention in the operating room anesthesia work area. Infection Control \& Hospital Epidemiology 2019; 40(1):1-17. doi:10.1017/ice.2018.303

8. Maslyk PA, et al. Microbial growth on the anesthesia machine. AANA J 2002;70(1):53-56.

9. Loftus RW, Brown JR, Koff MD, et al. Multiple reservoirs contribute to intraoperative bacterial transmission. Anesth \& Analg 2012;114(6):1236-48. doi: 10.1213/ ANE.0b013e31824970a2

10. Henry B, et al. An outbreak of Serratia marcescens associated with the anesthetic agent propofol. Am J Infect Control 2001;29(5):312-15. https://doi.org/10.1067/ mic.2001.117043
11. Lowman W, Veet al. Bacterial contamination of re-usablelaryngoscope blades during the course of daily anaesthetic practice. S Afr Med J 2013;103:386-89. http://dx.doi. org/10.7196/SAMJ.6385

12. Bhatt JM, et al. Microbiological sampling of the forgotten components of a flexible fiberoptic laryngoscope: what lessons can we learn? Otolaryngol Head Neck Surg 2014;150:235-36. https://doi. org/10.1177/0194599813513424

13. Munoz-Price LS, et al. Frequency of Interactions and Hand Disinfections among Anesthesiologists While Providing Anesthesia Care in the Operating Room: Induction versus Maintenance. Infection Control \& Hospital Epidemiology 2014; 35(8):1056-59. https://doi.org/10.1086/677154

14. Yokoe DS, et al. A compendium of strategies to prevent healthcare-associated infections in acute care hospitals: 2014 updates. Infect Control Hosp Epidemiol 2014;35:96777. https://doi.org/10.1017/S0899823X00193833

15. O'Grady NP, Alexander M, Burns LA, et al. Summary of recommendations: Guidelines for the Prevention of Intravascular Catheter-related Infections. Clin Infect Dis 2011;52:1087-99. https://doi.org/10.1093/cid/cir138 